\title{
THE CUMULANT REPRESENTATION OF THE LUNDBERG ROOT IN THE CASE OF SEMICONTINUOUS PROCESSES
}

UDC 519.21

\author{
D. V. GUSAK
}

\begin{abstract}
For the case of homogeneous processes $\xi(t), \xi(0)=0, t \geq 0$, with independent increments and negative jumps, it is proved in A. V. Skorokhod, Random Processes with Independent Increments, Nauka, Moscow, 1964 that the functional

$$
\tau^{+}(x)=\inf \{t \geq 0: \xi(t)>x\}, \quad x \geq 0,
$$

is a nondecreasing process with independent increments with respect to $x$, and its moment generating function is expressed via the cumulant that satisfies the corresponding Lundberg equation. The corresponding representations of this cumulant are specified and its Lévy characteristics (namely, $\gamma$ and Lévy's integral measure $N(x)$ ) are evaluated by using some of the results of the author's work of 2007 for the processes under consideration.
\end{abstract}

\section{INTRODUCTION}

Homogeneous processes $\xi(t), \xi(0)=0, t \geq 0$, with independent increments whose jumps are of a constant sign are called semicontinuous processes. In particular, if all the jumps of $\xi(t)$ are negative, then $\xi(t)$ is an upper continuous process and its LévyKhinchine cumulant is of the following form:

$$
\psi(\alpha)=\frac{1}{t} \ln \mathrm{E} e^{i \alpha \xi(t)}=i \alpha \gamma-\frac{\alpha^{2} \sigma^{2}}{2}+\int_{-\infty}^{0}\left(e^{i \alpha x}-1-\frac{i \alpha x}{1+x^{2}}\right) \Pi(d x)
$$

(see [1, §23]).

Now we introduce the functionals of interest:

$$
\begin{gathered}
\xi^{ \pm}(t)=\sup _{0 \leq t^{\prime} \leq t}(\inf ) \xi\left(t^{\prime}\right), \quad \xi^{ \pm}=\sup _{0 \leq t<\infty}(\inf ) \xi(t), \\
\tau^{+}(x)=\inf \{t>0: \xi(t)>x\}, \quad \tau^{-}(-x)=\inf \{t>0: \xi(t)<-x\}, \\
\gamma^{ \pm}( \pm x)=\xi\left(\tau^{ \pm}( \pm x)\right) \mp x, \quad x \geq 0 .
\end{gathered}
$$

We denote by $\theta_{s}$ a random variable whose distribution is exponential with parameter $s>0$, that is,

$$
\mathrm{P}\left\{\theta_{s}>t\right\}=e^{-s t}, \quad s, t>0 .
$$

Then the characteristic functions of $\xi(t)$ and $\xi\left(\theta_{s}\right)$ are expressed via the cumulant

$$
\psi(\alpha)=\ln \mathrm{E} e^{i \alpha \xi(1)}
$$

2010 Mathematics Subject Classification. Primary 60G50; Secondary 60K10.

Key words and phrases. Compound semicontinuous Poisson processes, semicontinuous homogeneous processes with independent increments, Lundberg's root. 
as follows:

$$
\mathrm{E} e^{i \alpha \xi(t)}=e^{t \psi(\alpha)}, \quad \varphi(s, \alpha)=: \mathrm{E} e^{i \alpha \xi\left(\theta_{s}\right)}=\frac{s}{s-\psi(\alpha)} .
$$

The equation

$$
k(r)=: \psi(-i r)=s, \quad s \geq 0,
$$

is called the fundamental Lundberg equation. If $\mathrm{E}|\xi(1)|^{2}<\infty$, then equation (3) has two roots $r_{1,2}(s)$, since $k(r)$ is convex in a neighborhood of zero in view of

$$
k^{\prime \prime}(0)=\operatorname{Var} \xi(1)>0 .
$$

We study the positive root $r_{2}(s)=\rho_{+}(s)$, called Lundberg's root, in order to obtain properties of the moment generating function of $\tau^{+}(x)$ and the characteristic function of $\xi^{+}\left(\theta_{s}\right)$, since they are uniquely determined by this root:

$$
\begin{gathered}
T(s, x)=\mathrm{E} e^{-s \tau^{+}(x)} \mathbb{1}_{\tau^{+}(x)<\infty}=e^{-x \rho_{+}(s)}, \quad x \geq 0, \\
\varphi_{+}(s, \alpha)=: \mathrm{E} e^{i \alpha \xi^{+}\left(\theta_{s}\right)}=\frac{\rho_{+}(s)}{\rho_{+}(s)-i \alpha} .
\end{gathered}
$$

If $m=\mathrm{E} \xi(1) \geq 0$, then $\rho_{+}(s) \rightarrow 0$ as $s \rightarrow 0, \mathrm{P}\left\{\tau^{+}(x)<\infty\right\}=1$, and

$$
T(s, x)=\mathrm{E} e^{-s \tau^{+}(x)}=\exp \left\{x\left[-s \gamma+\int_{0}^{\infty}\left(e^{-s y}-1\right) d N(y)\right]\right\}
$$

according to equality (23.2) of [1], where $\gamma \geq 0$ and $N(x)$ is a nondecreasing function such that $N(+\infty)=0$ and

$$
\int_{0}^{1} x d N(x)<\infty
$$

Thus equalities (44) and (6) imply that

$$
-\rho_{+}(s)=k_{T}(-s)=-s \gamma+\int_{0}^{\infty}\left(e^{-s y}-1\right) d N(y), \quad s>0 .
$$

This means that $-\rho_{+}(s)$ is the cumulant of the nondecreasing (with respect to $x$ ) process $T(x)=\tau^{+}(x)$ whose variation is bounded.

Our aim is to obtain more information about the Lévy characteristics $\gamma$ and $N(y)$ for the process $T(x)$. If the variation of $\xi(t)$ is unbounded, then one may expect that $\gamma=0$, while the properties of $N(x)$ are not known in this case.

\section{Semicontinuous process}

First we consider a compound Poisson process

$$
\xi(t)=a t-S(t), \quad a>0,
$$

where

$$
S(t)=\sum_{k \leq \nu(t)} \xi_{k}, \quad S(0)=0 .
$$

Here $\nu(t)$ is a simple Poisson process with intensity $\lambda>0$ and where the random variables $\xi_{k}$ are independent and identically distributed with a common distribution function

$$
F(x)=\mathrm{P}\left\{\xi_{k}<x\right\}, \quad x \geq 0,
$$

such that $F(0)=0$ and with the characteristic function

$$
\varphi(\alpha)=\mathrm{E} e^{i \alpha \xi_{k}}, \quad f(s)=\mathrm{E} e^{-s \xi_{k}}, \quad k \geq 1, \quad s \geq 0 .
$$


Compound Poisson processes have a wide range of applications in risk theory, queueing theory, etc. The characteristic function of $\xi(t)$ is determined by the cumulant

$$
\psi(\alpha)=i \alpha a+\lambda(\varphi(-\alpha)-1)
$$

(see equality (21)). Substituting $i \alpha=r$ we obtain

$$
k(r)=a r+\lambda(f(r)-1) .
$$

If $r=\rho_{+}(s)$ is the root of equation (3), then

$$
\rho_{+}(s)=\frac{s}{a}+\frac{\lambda}{a}\left[1-f\left(\rho_{+}(s)\right)\right] .
$$

For simplicity, consider the case of $a=1$. Then (8) describes a governing process of a queueing system. The nondecreasing processes

$$
\xi^{+}(t)=\alpha(t), \quad t \geq 0, \quad \text { with } \quad \alpha(0)=0
$$

and

$$
T(x)=\tau^{+}(x), \quad x \geq 0, \quad \text { with } \quad T(0)=0
$$

can be viewed as "inverses" to each other in a certain sense (see pp. 197-200 and the graphs represented on p. 176 in [2]). The process $\alpha(t)$ represents the idle period, while

$$
\beta(t)=t-\alpha(t)
$$

is the busy period. Clearly, $\alpha(t)$ is a continuous and stepwise linear function with respect to $t$; the lengths of the intervals where $\alpha(t)$ is constant are determined by the idle periods. The mirror image of the graph of $\xi^{+}(t)$ rotated counterclockwise about the origin by the angle $\frac{\pi}{4}$ represents the graph of the "inverse" process $T(x)=\tau^{+}(x)$. The intervals where $\alpha(t)$ and $T(x)$ grow linearly are the same, since $a=1$. We denote those intervals by $\tilde{\tau}_{r}$. The random variable $\tau_{k}$ being the time between two exponential jumps has an exponential distribution, as well. The random variable $\tilde{\tau}_{r}$ also has an exponential distribution with parameter $\lambda>0$.

The lengths of the intervals where $\alpha(t)$ is constant (as well as the value of $\alpha(t)$ on these intervals) are determined by the busy periods $\tilde{\theta}_{k}$. After the "inversion" described above, the lengths of the intervals $\tilde{\theta}_{k}$ determine the corresponding jumps of $T(x)$. The coefficients of the linear growth of $\alpha(t)$ and $T(x)$ are such that $a=1 / a=1$. Thus, similarly to (8), the stochastic process $T(x)$ admits the following stochastic representation:

$$
T(x)=x+\sum_{k \leq \tilde{\nu}(x)} \tilde{\theta}_{k}, \quad \tilde{\nu}(x)=\max \left\{n: \sum_{r \leq n} \tilde{\tau}_{r} \leq x\right\},
$$

where $\tilde{\nu}(x)$ (like $\nu(t))$ is a simple Poisson process with intensity $\lambda>0$.

By Theorem 4.10 (see [2, p. 198]), the moment generating function of the random variable $\tilde{\theta}_{r}$ is given by

$$
\pi(s)=\mathrm{E} e^{-s \tilde{\theta}_{1}} \mathbb{1}_{\tilde{\theta}_{1}<\infty}=f\left(\rho_{+}(s)\right)=\mathrm{E} e^{-s \tau^{+}\left(\xi_{1}\right)} .
$$

If $m=\mathrm{E} \xi(1) \geq 0$, then $\pi(s)=f\left(\rho_{+}(s)\right)=\mathrm{E} e^{-s \tilde{\theta}_{1}} \rightarrow 1$ and $\rho_{+}(s) \rightarrow 0$ as $s \rightarrow 0$. According to equality (9), the number $-\rho_{+}(s)$ is such that

$$
\begin{aligned}
& -\rho_{+}(s)=k_{T}(-s)=\ln \mathrm{E} e^{-s T(1)}=-s+\lambda(\pi(s)-1), \\
& T(s, x)=\mathrm{E} e^{-s T(x)}=e^{x k_{T}(-s)}, \quad x \geq 0 .
\end{aligned}
$$

This cumulant representation means that

$$
\gamma=1, \quad N(x)=-\lambda \mathrm{P}\left\{\tilde{\theta}_{1}>x\right\}, \quad x>0,
$$

in relation (7). 
D. V. GUSAK

If $m<0$, then $\rho_{+}(s) \rightarrow \rho_{+}>0$ and the conditional moment generating function of $T(x)$ is determined by

$$
\begin{gathered}
\hat{k}_{T}(-s)=\rho_{+}-\rho_{+}(s)=-s \lambda(\pi(s)-\pi(0)), \quad \pi(0)=f\left(\rho_{+}(s)\right)<1, \\
\hat{T}(s, x)=\mathrm{E}\left[e^{-s T(x)} / T(x)<\infty\right]=e^{x \hat{k}_{T}(-s)}, \quad x \geq 0 .
\end{gathered}
$$

Similarly to the stochastic process (8), the following result obtains a more general relation for an arbitrary drift $a>0$. We also determine the Lévy characteristics of the process $T(x)$.

Theorem 1. Let an upper continuous process (8) have a positive drift a $>0$ and let

$$
\lambda=\int_{-\infty}^{0} \Pi(d x)<\infty
$$

If $m \geq 0$, then the moment generating function of $T(x)=\tau^{+}(x), x \geq 0$, with the corresponding cumulant $k_{T}(-s)=-\rho_{+}(s)$ is uniquely determined by the root of equation (3) as follows:

$$
\begin{gathered}
T(s, x)=\mathrm{E} e^{-s T(x)} \mathbb{1}_{T(x)<\infty}=\mathrm{E} e^{-s T(x)}=e^{x k_{T}(-s)}, \\
k_{T}(-s)=-\frac{s}{a}+\frac{\lambda}{a}(\pi(s)-1), \quad \pi(s)=\mathrm{E} e^{-s \tau^{+}\left(\xi_{1}\right)}=f\left(\rho_{+}(s)\right) .
\end{gathered}
$$

In particular, the Lévy characteristics in (7) are such that

$$
\gamma=\frac{1}{a}, \quad N(x)=-\frac{\lambda}{a} \mathrm{P}\left\{\tilde{\theta}_{1}>x\right\}, \quad \tilde{\theta}_{1} \doteq \tau^{+}\left(\xi_{1}\right), \quad x>0 .
$$

If $m<0$, then $\rho_{+}(s) \rightarrow \rho_{+}>0$ as $s \rightarrow 0$ and the distribution of $T(x)$ is improper and

$$
\mathrm{P}\{T(x)<\infty\}=e^{-x \rho_{+}}<1 .
$$

The conditional moment generating function of $T(x)$ is given by relation (14), and the cumulant is

$$
\begin{aligned}
\hat{k}_{T}(-s) & =\rho_{+}-\rho_{+}(s)=-\frac{s}{a}+\frac{\lambda}{a} \int_{0}^{\infty}\left(e^{-s y}-1\right) d \mathrm{P}\left\{\tilde{\theta}_{1}<y, \tilde{\theta}_{1}<\infty\right\} \\
& =-\frac{s}{a}+\frac{\lambda}{a}(\pi(s)-\pi(0)), \quad \pi(0)=f\left(\rho_{+}\right)<1 .
\end{aligned}
$$

Proof. Similarly to the proof for the case of $a=1$, we use the following cumulant representation:

$$
k(r)=: a r+\lambda(f(r)-1)=s
$$

for equation (3). Substituting $r=\rho_{+}(s)>0$ in the latter equation with $m \geq 0$ we derive representations (15) and (16) for the cumulant $k_{T}(-s), s \geq 0, k(0)=0$, and for its Lévy characteristics.

If $m<0$, then the random variable $T(x)$ has an improper distribution, namely

$$
\lim _{s \rightarrow 0} T(s, x)=\lim _{s \rightarrow 0} \mathrm{E} e^{-s T(x)} \mathbb{1}_{T(x)<\infty}=\mathrm{P}\left\{\tau^{+}(x)<\infty\right\}=e^{-\rho_{+} x}<1 .
$$

Thus one can consider the conditional moment generating function

$$
\hat{T}(s, x)=T(s, x) / \mathrm{P}\{T(x)<\infty\}=e^{x\left(\rho_{+}-\rho_{+}(s)\right)}
$$

instead of $T(s, x)$, where the cumulant $\hat{k}_{T}(-s)=\rho_{+}-\rho_{+}(s)$ admits the representation (17). 


\section{Almost semicontinuous process}

Consider an almost upper semicontinuous process with the cumulant

$$
\psi(\alpha)=\frac{\lambda_{1} c}{c-i \alpha}+i \alpha a+\int_{-\infty}^{0}\left(e^{i \alpha x}-1\right) \Pi(d x), \quad a \leq 0, c, \lambda_{1}>0 .
$$

This process can be represented as a difference of two nondecreasing processes

$$
\xi(t)=\xi_{1}(t)-\xi_{2}(t)
$$

The corresponding cumulants of $\xi_{1}$ and $\xi_{2}$ are given by

$$
\begin{gathered}
\psi_{1}(\alpha)=\frac{\lambda_{1} c}{c-i \alpha}, \\
\psi_{2}(\alpha)=i \alpha|a|-\int_{0}^{\infty}\left(e^{-i \alpha x}-1\right) \Pi(-d x), \quad \int_{0}^{1} x \Pi(-d x)<\infty,
\end{gathered}
$$

respectively.

The trajectories of the stochastic process $\xi(t)$ are stepwise linear functions with the drift $a<0$. The trajectories of the process $\alpha(t)=\xi^{+}(t)$ are nondecreasing stepwise constant functions. The intervals where $\alpha(t)$ is constant, $\alpha(0)=0$, are determined by the random variables $\tilde{\theta}_{k}=\tau^{+}(0)$. The same random variables $\tilde{\theta}_{k}$ determine the values of the process $\alpha(t)$ in these intervals. The jumps of $\alpha(t)$ are defined by the excesses $\gamma_{k}^{+}$ related to the processes $\xi(t)$. The random variables $\gamma_{k}^{+}$have the exponential distribution with parameter $c>0$.

The heights of the jumps of the "inverse" process $T(x)$ are determined by the random variables $\tilde{\theta}_{k}$, while the lengths of the intervals where $T$ is constant are determined by the random variables $\gamma_{k}^{+}$. Thus

$$
T(x)=\sum_{0 \leq k \leq \tilde{\nu}_{c}(x)} \tilde{\theta}_{k}, \quad \tilde{\nu}_{c}(x)=\max \left\{n: \sum_{k \leq n} \gamma_{k}^{+} \leq x\right\},
$$

where $\tilde{\nu}_{c}(x)$ is a simple Poisson process with intensity $c>0$.

Using the stochastic relation (19) one can easily obtain the following result.

Theorem 2. Let $\xi(t)$ be an upper almost semicontinuous process with cumulant (18). Then the stochastic process $T(x)=\tau^{+}(x), T(0)=\tau^{+}(0)>0$, is a nondecreasing homogeneous process admitting the stochastic representation (19) if $m \geq 0$. The cumulant and moment generating function of the process $T_{0}(x)=\tau^{+}(x)-\tau^{+}(0), T_{0}(0)=0$, are determined by the Lundberg root $\rho_{+}(s)>0$ of equation (3), namely

$$
\begin{gathered}
k_{T_{0}}(-s)=-\rho_{+}(s)=c(\pi(s)-1), \quad \pi(s)=\mathrm{E} e^{-s \tau^{+}(0)}=q_{+}(s), \\
\quad \lim _{s \rightarrow 0} q_{+}(s)=1 ; \\
T_{0}(s, x)=\mathrm{E} e^{-s T_{0}(x)}=e^{x k_{T}(-s)}, \quad x \geq 0 .
\end{gathered}
$$

If $m<0$, then $\lim _{s \rightarrow 0} \rho_{+}(s)=\rho_{+}>0$ and the conditional moment generating function $T_{0}(x)$ is determined from the following relation:

$$
\begin{aligned}
& \hat{T}_{0}(s, x)=\mathrm{E}\left[e^{-s T_{0}(x)} / T_{0}(x)<\infty\right]=e^{x \hat{k}_{T_{0}}(-s)}, \quad x \geq 0, \\
& \hat{k}_{T_{0}}(-s)=\rho_{+}-\rho_{+}(s)=c(\pi(s)-\pi(0)), \quad \pi(0)=q_{+}(0)<1 .
\end{aligned}
$$

Relations (20) and (21) imply that

$$
\gamma=0, \quad d N(y)= \begin{cases}c d \mathrm{P}\left\{\tau^{+}(0)<y\right\}, & m \geq 0, y>0 \\ c d \mathrm{P}\left\{\tau^{+}(0)<y, \tau^{+}(0)<\infty\right\}, & m<0 .\end{cases}
$$


Example 1. Let $\xi(t)$ be an upper almost semicontinuous and at the same time lower almost semicontinuous symmetric stochastic process (see Example 2.4 in 2 for $m=0$ ) with the cumulant $\psi(\alpha)=\lambda(i \alpha)^{2} /\left(c-(i \alpha)^{2}\right)$. Then

$$
\rho_{+}(s)=c p_{+}(s), \quad p_{+}(s)=\mathrm{P}\left\{\tau^{+}(0)<\theta_{s}\right\}=\mathrm{P}\left\{\xi^{+}\left(\theta_{s}\right)=0\right\}=\sqrt{\frac{s}{s+\lambda}} .
$$

Inverting $p_{+}(s)$ with respect to $s$, we obtain from (22)

$$
\begin{aligned}
N(z) & =-\frac{2 c}{\pi} \int_{0}^{1} e^{-\lambda z y} d \arcsin \sqrt{y}, \quad N(0)=-c, z \geq 0 \\
d N(z) & =\frac{\lambda c}{\pi} \int_{0}^{1} \sqrt{\frac{y}{1-y}} e^{-\lambda z y} d y, \quad z>0 .
\end{aligned}
$$

Denote by $Q_{0, w}(t)$ the time spent by the standard Wiener process $w(u), u \in \mathbb{R}^{+}$, in the interval $0 \leq u \leq t$. Then

$$
|N(t)|=c \mathrm{E} e^{-\lambda Q_{0, w}(t)}, \quad t>0 .
$$

\section{Semicontinuous PRocess With Jumps Whose Intensity is Unbounded}

Now we consider an upper semicontinuous process $\xi(t)$ instead of the process defined by (8). We assume that

$$
\int_{-\infty}^{0} \Pi(d x)=\infty
$$

and that its variation is bounded. Then the cumulant is given by

$$
\begin{gathered}
\psi(\alpha)=i \alpha a+\int_{-\infty}^{0}\left(e^{i \alpha x}-1\right) \Pi(d x), \quad a>0, \\
\int_{-1}^{0}|x| \Pi(d x)<\infty .
\end{gathered}
$$

Since $a>0$, there are intervals where the trajectories of $\xi(t)$ and $\xi^{+}(t)$ grow linearly with the drift $a^{-1}$ (moreover there are intervals of arbitrary small lengths, since $\lambda=\infty$ ).

Denoting $i \alpha=r$, we represent the cumulant $k(r)$ as follows:

$$
k(r)=r a+\int_{0}^{\infty}\left(e^{-r x}-1\right) \Pi(-d x) .
$$

Using equality (3) and relations (44) and (25) we obtain the following result.

Theorem 3. Let a process $\xi(t)$ be upper continuous and let its cumulant be given by equalities (24)-(25). If $m \geq 0$, then the cumulant of the homogeneous monotonic process $T(x)$ is defined by equality (7), while its Lévy characteristics are given by

$$
\gamma=\frac{1}{a}, \quad N(y)=-\frac{1}{a} \int_{0}^{\infty} \mathrm{P}\left\{\tau^{+}(x)<y\right\} \Pi(-d x), \quad y>0 .
$$

If $m<0$, then

$$
\lim _{s \rightarrow 0} \rho_{+}(s)=\rho_{+}>0, \quad T(s, 0)=\mathrm{P}\left\{\xi^{+}\left(\theta_{s}\right)>0\right\}=q_{+}(s)>0,
$$

and the conditional moment generating function of $T(x)$ is determined from the relation

$$
\hat{T}(s, x)=\mathrm{E}\left[e^{-s T(x)} / T(x)<\infty\right]=e^{x\left(\rho_{+}-\rho_{+}(s)\right)}=e^{x \hat{k}_{T}(-s)}, \quad x \geq 0,
$$

where

$$
\hat{k}_{T}(-s)=-\frac{s}{a}+\frac{1}{a} \int_{0}^{\infty}\left(e^{-s y}-1\right) \int_{0}^{\infty} \Pi(-d x) d \mathrm{P}\left\{\tau^{+}(x)<y, \tau^{+}(x)<\infty\right\} .
$$


Proof. The Lundberg equation (3) implies that

$$
-a \rho_{+}(s)=\int_{0}^{\infty}\left(e^{-\rho_{+}(s) x}-1\right) \Pi(-d x)-s .
$$

If $m \geq 0$, then

$$
\mathrm{E} e^{-s \tau^{+}(x)}=e^{-\rho_{+}(s) x}=\int_{0}^{\infty} e^{-s y} d \mathrm{P}\left\{\tau^{+}(x)<y\right\}, \quad x>0 .
$$

Thus (28) implies a "cumulant" relation for $k_{T}(-s)=-\rho_{+}(s)$, namely

$$
-\rho_{+}(s)=-\frac{s}{a}+\frac{1}{a} \int_{0}^{\infty}\left(e^{-s y}-1\right) \int_{0}^{\infty} d \mathrm{P}\left\{\tau^{+}(x)<y\right\} \Pi(-d x) .
$$

Comparing equalities (28) and (7) we easily derive relation (26) for Lévy's characteristics $\gamma$ and $N(y)$.

Relation (27) can be obtained similarly for the conditional moment generating function $\hat{T}(s, x)$ with the cumulant $\hat{k}_{T}(-s)$ if $m<0$ and the Lévy measure is such that

$$
d N(y)=\frac{1}{a} \int_{0}^{\infty} d \mathrm{P}\left\{\tau^{+}(x)<y, \tau^{+}(x)<\infty\right\} \Pi(-d x), \quad y>0 .
$$

\section{BROWNIAN MOTION}

It is shown in 3 that $T(x)=\tau^{+}(x), x>0$, is a stable process with respect to $x$ of index $\alpha_{*}=1 / 2$ if the process $\xi(t)$ is Brownian motion. Below we prove a more general statement for Brownian motion.

Theorem 4. Let $\xi_{0}(t)=a t+\sigma w(t)$, where $\sigma>0$. The moment generating function of $T(x)=\tau^{+}(x)=\inf \left\{t>0: \xi_{0}(t)>x\right\}$ for $a \geq 0$ is such that

$$
\begin{gathered}
T(s, x)=\mathrm{E} e^{-s \tau^{+}(x)}=e^{x k_{T}(-s)}, \quad x \geq 0, \\
k_{T}(-s)=-\rho_{+}(s)=\int_{0}^{\infty}\left(e^{-s x}-1\right) d N_{a}(x) .
\end{gathered}
$$

If $a=0$, then

$$
N_{0}(x)=-\frac{2}{\sigma} \frac{1}{\sqrt{2 \pi x}}=-\frac{1}{\sigma} \sqrt{\frac{2}{\pi x}}, \quad x>0,
$$

and

$$
N_{a}(x)=N_{0}(x) \exp \left\{-\frac{a^{2} x}{2 \sigma^{2}}\right\}+\frac{2 a}{\sigma^{2}} \Phi_{0}\left(\frac{a \sqrt{x}}{\sigma}\right), \quad a>0,
$$

where

$$
\Phi_{0}(x)=\frac{1}{2 \pi} \int_{x}^{\infty} e^{-z^{2} / 2} d z, \quad x>0 .
$$

If $a<0$, then the conditional moment generating function of $T(x)$ is determined from the following relation:

$$
\hat{T}(s, x)=\mathrm{E}\left[e^{-s T(x)} / T(x)<\infty\right]=e^{x \hat{k}_{T}(-s)}, \quad x \geq 0,
$$

and the cumulant is such that

$$
\begin{gathered}
\hat{k}_{T}(-s)=\rho_{+}-\rho_{+}(s)=\int_{0}^{\infty}\left(e^{-s x}-1\right) d \hat{N}_{a}(x), \\
\hat{N}_{a}(x)=N_{0}(x) e^{-a^{2} x /\left(2 \sigma^{2}\right)}+\frac{2|a|}{\sigma^{2}} \Phi_{0}\left(\frac{|a| \sqrt{x}}{\sigma}\right), \quad x>0 .
\end{gathered}
$$


Proof. The Lundberg roots $\pm \rho_{ \pm}(s)$ of the equation

$$
\begin{gathered}
2 a r+\sigma^{2} r^{2}=2 s, \\
\rho_{+}(s)=\frac{1}{\sigma^{2}}\left(\sqrt{a^{2}+2 s \sigma^{2}} \mp a\right)>0
\end{gathered}
$$

uniquely determine the moment generating functions for $\tau^{ \pm}( \pm x), x>0$. We consider separately the following two cases:

(1) Case of $a \geq 0$. Then the positive root admits the representation

$$
-\rho_{+}(s)=-\frac{2 s}{a+\sqrt{a^{2}+2 s \sigma^{2}}} \underset{s \rightarrow 0}{\longrightarrow} 0 .
$$

(2) Case of $a<0$. Then

$$
\rho_{+}(s) \underset{s \rightarrow 0}{\longrightarrow} \rho_{+}>0
$$

and the above representation holds for

$$
\rho_{+}-\rho_{+}(s)=\hat{k}_{T}(-s)=-\frac{2 s}{|a|+\sqrt{a^{2}+2 s \sigma^{2}}} \underset{s \rightarrow 0}{\longrightarrow} 0 .
$$

Since the dependence of $k_{T}(-s)$ and $\hat{k}_{T}(-s)$ on $s$ in (35) and (36), respectively, is the same, the right-hand sides of (32) and (34) are similar with respect to $x$ (for $a>0$ and $a<0$, respectively).

First we consider the case of $a \geq 0$. The Lévy characteristics of the process $\xi_{0}(t)$ in (7) are determined by the conditions $\gamma=0$ and $\int_{0}^{\infty} x d N_{a}(y)<\infty$, whence

$$
\rho_{+}(s)=\int_{0}^{\infty}\left(1-e^{-s y}\right) d N_{a}(y)=s \int_{0}^{\infty}\left|N_{a}(y)\right| e^{-s y} d y .
$$

Putting $\tilde{n}_{a}(s)=\int_{0}^{\infty}\left|N_{a}(x)\right| e^{-s x} d x$ we obtain

$$
\rho_{+}(s)=s \tilde{n}_{a}(s), \quad \tilde{n}_{a}(s)=\left(\frac{a}{2}+\sqrt{\frac{a^{2}}{4}+\frac{\sigma^{2} s}{2}}\right)
$$

in view of equality (34).

Denoting $p=a^{2} / 4+s \sigma^{2} / 2$ and $s=2 p / \sigma^{2}+a^{2} /\left(2 \sigma^{2}\right)$, the Laplace transform of $\left|N_{a}(x)\right|$ is reduced to the following function:

$$
\tilde{f}(p)=\frac{1}{c+\sqrt{p}}=\int_{0}^{\infty} e^{-p x} f(x) d x .
$$

Inverting (38) with respect to $p$ (by using the tables of the Laplace transform (see [5, p. 210])) we find

$$
f(x)=\frac{1}{\sqrt{\pi x}}-a e^{-a^{2} x / 4} \Phi_{0}\left(a \sqrt{\frac{x}{2}}\right), \quad x>0 .
$$

Using the notation $s=2 p / \sigma^{2}+a^{2} /\left(2 \sigma^{2}\right)$ again we obtain

$$
\tilde{n}_{a}(s)=\int_{0}^{\infty}\left|N_{a}(x)\right| \exp \left\{-\frac{2 p x}{\sigma^{2}}+\frac{a^{2} x}{2 \sigma^{2}}\right\} d x .
$$

For $y=2 x / \sigma^{2}$, the right-hand side of (38) becomes of the following form:

$$
\frac{\sigma^{2}}{2} \int_{0}^{\infty}\left|N_{a}\left(\frac{\sigma^{2} y}{2}\right)\right| e^{a^{2} y / 4} e^{-p y} d y=\tilde{f}(p) .
$$


This together with (39) implies that

$$
\begin{gathered}
\frac{\sigma^{2}}{2}\left|N_{a}\left(\frac{\sigma^{2} y}{2}\right)\right| e^{a^{2} y / 4}=\frac{1}{\sqrt{\pi y}}-a e^{a^{2} y / 4} \Phi_{0}\left(a \sqrt{\frac{y}{2}}\right), \\
\left|N_{0}\left(\frac{\sigma^{2} y}{2}\right)\right|=\frac{2}{\sigma^{2}} \frac{1}{\sqrt{\pi y}}, \quad y>0, a=0, \\
\left|N_{a}\left(\frac{\sigma^{2} y}{2}\right)\right|=\frac{2}{\sigma^{2}} \frac{1}{\sqrt{\pi y}} e^{-a^{2} y / 4}-\frac{2 a}{\sigma^{2}} \Phi_{0}\left(a \sqrt{\frac{y}{2}}\right), \quad a>0 .
\end{gathered}
$$

Turning back to the variable $x=\sigma^{2} y / 2$, we prove both equalities (31) and (32). Note that if $a=0$, then

$$
\left|N_{0}(x)\right|=\int_{x}^{\infty} d N(d y)=\frac{1}{\sigma} \sqrt{\frac{2}{\pi x}}, \quad x \geq 0,
$$

is the integral Lévy measure of jumps of a stable process with index $\alpha_{*}=1 / 2$. If $a \neq 0$, then the measure $\left|N_{a}(x)\right|=\int_{x}^{\infty} d N_{a}(y)$ is expressed in terms of $\left|N_{0}(x)\right|$ and of the tails of the normal distribution (see (32) and (34)).

If $a<0$, then $\rho_{+}(s) \rightarrow \rho_{+}$and $T(x)$ has an improper distribution, since

$$
\mathrm{P}\left\{\tau^{+}(x)<\infty\right\}=\mathrm{P}\left\{\xi^{+}>x\right\}=e^{-\rho_{+} x}<1, \quad x<0 .
$$

As above, the conditional moment generating function of the random variable $T(x)$ is exponential, namely

$$
\hat{T}(s, x)=\frac{T(s, x)}{\mathrm{P}\{T(x)<\infty\}}=e^{\hat{k}_{T}(-s) x},
$$

where the cumulant $\hat{k}_{T}(-s)=\rho_{+}-\rho_{+}(s)$ admits representation (36) similar to (35). Equality (36) implies that $\hat{N}_{a}(x)$ in (34) can be obtained from (42)-(44) if $a$ is changed by $|a|$. Thus the theorem is proved.

Example 2. Let $\xi(t)=\xi_{*}(t)-S(t)$, where $\xi_{*}(t)$ is a stable process with index $\alpha_{*}=1 / 2$ and negative jumps $\left(c_{2}=0, c_{1}>0\right.$; see [1, p. 145]), and let $S(t)=\sum_{k \leq \nu(t)} \xi_{k}$ be a compound Poisson process with positive exponential jumps $\xi_{k}>0$. Then the cumulant of $\xi(t)$ is of the following form:

$$
\begin{gathered}
\psi(\alpha)=\frac{i \alpha}{c-i \alpha}-2 C|\alpha|^{1 / 2}, \quad C=4 c_{1} \sqrt{\pi}, \quad c, c_{1}>0, \\
k(r)=\psi(-i r)=\frac{r}{c-r}-2 C \sqrt{|r|} .
\end{gathered}
$$

The process $\xi(t)$ is upper almost semicontinuous and one can easily evaluate $\varphi_{+}(s, \alpha)$, $T(s, x), m \geq 0$, and $\hat{T}(s, x), m<0$, if the root $\rho_{+}(s)$ of equation (3) is known.

The root $\rho_{+}(s)$ exists if $s=0$. This can be proved by considering the following two functions:

$$
y_{1}=\frac{r}{c-r}, \quad y_{2}=2 C \sqrt{r} .
$$

The graphs of $y_{1}$ and $y_{2}$ intersect if $0<r<c$ and the intersection point determines the root $\rho_{+}>0$.

Similarly, if $s>0$ is sufficiently small, the root $\rho_{+}(s)>0$ of equation (3) is determined by the intersection point of the graphs of functions:

$$
y_{1}=\frac{r}{c-r}-s, \quad 0 \leq r<c, \quad y_{2}=2 C \sqrt{r} .
$$

Lemma 3.3 in [2] implies that the root $\rho_{+}(s)=c p_{+}(s) \leq c$ determines

$$
\varphi_{+}(s, \alpha)=\frac{p_{+}(s)(c-i \alpha)}{\rho_{+}(s)-i \alpha}, \quad T(s, x)=q_{+}(s) e^{-\rho_{+}(s) x}, \quad x \geq 0 .
$$


Note that $m=-\infty<0$ in the case under consideration. Thus we consider its conditional moment generating function $T_{0}(s, x)$ that, according to (21), can be rewritten in the following exponential form:

$$
\hat{T}_{0}(s, x)=\mathrm{E}\left[e^{-s\left(\tau^{+}(x)-\tau^{+}(0)\right)} / \tau^{+}(x)-\tau^{+}(0)<\infty\right]=e^{x \hat{k}_{T_{0}}(-s)} .
$$

Moreover

$$
\hat{k}_{T_{0}}(-s)=\rho_{+}-\rho_{+}(s)=c(\pi(s)-\pi(0)),
$$

where

$$
\pi(s)=\mathrm{E} e^{-s \tau^{+}(0)} \mathbb{1}_{\tau^{+}(0)<\infty}, \quad \pi(0)=q_{+}<1 .
$$

Note that the root $\rho_{+}(s)>0$ of equation (3) plays a crucial role only in the case of semicontinuous and of almost semicontinuous processes (see equalities (4) -(5)). For other processes, equation (3) may not have roots. Even though the roots exist, they do not uniquely determine the moment generating function $T(s, x)$ and characteristic function $\varphi_{+}(s, \alpha)$.

\section{BIBLIOGRAPHY}

1. A. V. Skorokhod, Random Processes with Independent Increments, Nauka, Moscow, 1964; English transl., Kluwer Academic Publishers Group, Dordrecht, 1991. MR.1155400 (93a:60114)

2. D. V. Gusak, Boundary Problems for Processes with Independent Increments in Risk Theory, Trans. Institute of Mathematics, National Academy of Sciences of Ukraine, vol. 67, Kiev, 2007.

3. P. Lévy, Processus Stochastiques et Mouvement Brownien, Gauthier-Villars, Paris, 1948. MR 0190953 (32:8363)

4. A. Erdelyi, W. Magnus, F. Oberhettinger, and F. G. Tricomi, Tables of integral transforms, vol. 1, McGraw-Hill Book Company, Inc., New York-Toronto-London, 1954. MR0061695 $(15: 868 \mathrm{a})$

5. G. Korn and T. Korn, Mathematical Handbook for Scientists and Engineers, Second, enlarged and revised edition, McGraw-Hill Book Co., New York-Toronto-London, 1968. MR0220560 $(36: 3618)$

Institute for Mathematics, National Academy of Sciences of Ukraine, Tereshchenkivs'ka Street 3, Kyiv-4 252601, Ukraine

E-mail address: random@imath.kiev.ua

Received 19/OCT/2009

Translated by OLEG KLESOV 